\title{
Stem Cells for Extreme Prematurity
}

\author{
Bernard Thébaud, MD, PhD ${ }^{1,2,3,4}$ \\ ${ }^{1}$ Sinclair Centre for Regenerative Medicine, Ottawa Hospital Research \\ Institute, Ottawa, Ontario, Canada \\ 2 Department of Molecular Medicine, Children's Hospital of Eastern \\ Ontario Research Institute, Ottawa, Ontario, Canada \\ ${ }^{3}$ Department of Cellular and Molecular Medicine, University of \\ Ottawa, Ottawa, Ontario, Canada \\ ${ }^{4}$ Division of Neonatology, Department of Pediatrics, Children's \\ Hospital of Eastern Ontario, Ottawa, Ontario, Canada \\ Am J Perinatol 2019;36(suppl S2):S68-S73.
}

\begin{abstract}
Address for correspondence Bernard Thébaud, MD, PhD, Sinclair Centre for Regenerative Medicine, Ottawa Hospital Research Institute, 501 Smyth Road, Ottawa, ON K1H 8L6, Canada (e-mail: bthebaud@toh.on.ca).
\end{abstract}

\author{
Abstract \\ Keywords \\ - lung \\ - injury \\ - stem cells \\ - regenerative \\ medicine \\ - preterm birth
}

Regenerative medicine is a bourgeoning field promising to repair damaged organs and thus has created high hopes in neonatology to curb some of the complications due to extreme preterm birth. Extensive laboratory investigations over the past 15 years have tried to harness the regenerative potential of a variety of (stem) cell-based therapies. Most preclinical studies have focused on experimental neonatal lung and brain injury. These promising results lead to the initiation of phase I clinical trials for chronic lung disease of prematurity and severe intraventricular hemorrhage, two of the most devastating complications of extreme preterm birth. Despite this relative rapid clinical translation, major gaps persist in our understanding of the biology of these putative repair cells and our ability to predict the quality and thus the efficacy of the cell product. This review will provide a brief overview of the various cell-based therapies that have been investigated in experimental neonatal lung injury and the remaining challenges in utilizing these new, disruptive therapies to their full extend to realize the promise of regenerative medicine in neonatology.
Bronchopulmonary dysplasia (BPD), the chronic lung disease of prematurity, remains the main complication of extreme prematurity. ${ }^{1}$ Dramatic improvements in perinatal care have substantially increased neonatal survival from 34 weeks' gestation since the original description of BPD in $1967^{2}$ to 22 to 24 weeks' gestation nowadays. The ransom of success is the increasing challenge of protecting an ever more immature and fragile lung from the multiple deleterious effects of inflammation, oxidative stress, mechanical ventilation, and suboptimal growth. ${ }^{3}$ Even so the lung has robust repair potential, the life-long consequences of interfering with early lung development remain unknown. Increasing evidence in the literature suggests reduced lung function, airway hyperreactivity, ${ }^{4}$ and impaired lung vascular growth, ${ }^{5}$ as well as other organ impairment ${ }^{6}$ justifying the need for exploring effective early interventions that are currently lacking.

Recent insights into the regenerative potential of stem cells ${ }^{7}$ have created excitement in neonatology for the treatment of complications of extreme prematurity including BPD. Stem cells are defined as cells capable of self-renewal and differ- entiation into at least one other cell type. However, it is not these fundamental properties that seem to underlie their mechanism of action. Preclinical and some clinical evidence do not support the local proliferation and differentiation of these cells into lung cells for repair. The therapeutic benefits seem to emanate from a by-stander effect: the secretion of repair modulating factors in the microenvironment, ${ }^{8}$ cell-tocell interactions via the exchange of micro-organelles, and release of extracellular vesicles (EVs). ${ }^{9}$ Given the excitement in the field, a panoply of cell-based therapies have emerged.

\section{Brief Appraisal of Cell-Based Therapies Investigated in Preclinical and Clinical Studies of Neonatal Lung Injury}

\section{Mesenchymal Stromal Cells-The Front Runner}

Definition

Mesenchymal stromal cells (MSCs) are plastic adherent, multipotent cells (they can be induced to become cartilage, bone, or
Copyright $\odot 2019$ by Thieme Medical Publishers, Inc., 333 Seventh Avenue, New York, NY 10001, USA. Tel: +1(212) 584-4662. ISSN 0735-1631. 
fat) that express certain cell surface markers (CD105, CD73 and CD90, and lack of CD45, CD34, CD14 or CD11b, CD79 $\alpha$ or CD19 and HLA-DR surface molecules) as defined by the International Society for Cellular Therapy. ${ }^{10}$ Originally described in the bone marrow, ${ }^{11}$ MSCs have now been shown to exist in all organs including the umbilical cord tissue and cord blood. ${ }^{12}$

\section{Rationale and Biological Plausibility}

MSCs are potent immune-modulatory cells, but also exert pleiotropic effects that target numerous other pathophysiological mechanisms that contribute to BPD: MSCs are antifibrotic, antioxidant, and proangiogenic. ${ }^{8}$ Other logistical aspects make them appealing as an off-the-shelf ready-touse cell therapy product as MSCs express HLA class I, but not HLA class II, and thus do not elicit alloreactive lymphocyte proliferation, enabling allogeneic transplantation of MSCs. Furthermore, evidence suggests that MSCs are more abundant in the tracheal aspirate of preterm infants that go on to develop BPD versus those that do not. ${ }^{13}$ These MSCs seem to acquire by default a profibrotic phenotype suggesting they may lose their repair potential and/or even contribute to the disease pathogenesis. These findings are corroborated by observations of human fetal lung mesenchymal cells exposed to hyperoxia in vitro ${ }^{14}$ and in resident lung MSCs isolated from neonatal rats exposed to 14 days of hyperoxia. ${ }^{15}$

\section{Preclinical Evidence}

Numerous investigators have demonstrated the lung protective effect of MSCs derived from rat bone marrow ${ }^{16,17}$ and human umbilical cord tissue ${ }^{18}$ and cord blood ${ }^{19}$ in the neonatal rodent model exposed to hyperoxia. Even so various endpoints were assessed including the route of administration, dose-response, timing, long-term effect, and toxicity, as well as some mechanisms of action, most of these studies were exploratory in nature. While a systematic review and meta-analysis of all preclinical studies with MSCs in experimental neonatal lung injury confirmed the therapeutic benefit of MSCs on alveolarization, inflammation, and lung vascular growth, it also revealed some limitations in experimental design, reporting, and risk of bias, as well as the lack of studies in large animals. ${ }^{20}$

\section{Clinical Trials}

Nonetheless, the promising preclinical data and the apparent safety and lack of engraftment of MSCs have encouraged early phase clinical trials in preterm infants at risk of developing BPD. The first phase I clinical trial tested allogeneic cord bloodderived MSCs in nine preterm neonates born between 23 and 29 weeks' gestational age at 5 to 14 days of life if still on mechanical ventilation. ${ }^{21} \mathrm{~A}$ single intratracheal injection of $10^{7}$ or $2 \times 10^{7}$ MSCs was shown to be feasible and well tolerated without serious adverse events or dose-limiting toxicity. At 2 years follow-up, there were no adverse effects on growth, respiratory, and neurodevelopmental outcomes. ${ }^{22}$ Results of a U.S. trial with the same cell product and similar design are pending (NCT02381366). A phase II randomized, doubleblinded, multicenter, controlled trial using a low dose is currently underway (NCT01828957). A recent case report of two preterm infants with severe BPD treated at late stages of the disease showed that the repeated intravenous (IV) injection of bone marrow-derived MSCs was feasible and safe, but it did not influence disease outcome. ${ }^{23}$ Postmortem lung analysis confirmed absence of lung engraftment of transplanted cells. Welldesigned early phase trials over the coming years will provide more information regarding feasibility and safety of routes of administration, timing, and repeated administration. ${ }^{24}$

\section{Human Amniotic Epithelial Cells-The Mimic: Different Cell Type, Same Mechanism of Action}

\section{Definition}

A large number of human amniotic epithelial cells (hAECs) can be isolated from the amniotic membrane of the placenta after birth. hAECs are stem cell-like cells with self-renewal and multilineage potential. ${ }^{25}$ Similar to MSCs, they have limited class IA and II HLA expression and thus present a low risk of allogeneic rejection.

\section{Rationale and Biological Plausibility}

hAECs also share similar pleiotropic, mostly anti-inflammatory, but also proangiogenic, properties with MSCs. ${ }^{25}$ hAECs do not engraft and exert their therapeutic benefit through a paracrine activity.

\section{Preclinical Evidence}

Extensive experimental evidence has demonstrated the lung protective effects of hAECs not only in neonatal rodents exposed to hyperoxia, but also in fetal sheep exposed in utero to lipopolysaccharide or mechanical ventilation. ${ }^{26-28}$ Similar to MSCs, the relative ease of cell isolation, expansion and the lack of risk of rejection, and apparent safety profile are appealing characteristics for an off-the-shelf cell product.

\section{Clinical Trials}

Given the promising preclinical data, a phase I trial was recently conducted in six preterm infants born at less than 29 weeks' gestation with established severe BPD receiving a single IV infusion of 1 million hAECs $/ \mathrm{kg} .{ }^{29}$ An important lesson from this trial was the adjustment of the infusion protocol after the first infant experienced transient bradycardia and hypoxia during the infusion. This was likely related to a pulmonary microembolic event and did not reoccur in the remaining five infants after changes were made to the cell infusion protocol. This trial showed feasibility and lack of toxicity of a single IV dose of hAECs. The same group is now launching a phase I trial to test the feasibility and safety of a dose escalation $(2,10$, and 30 million cells $/ \mathrm{kg}$ ) in 24 preterm infants at high risk of severe BPD still requiring an $\mathrm{FiO}_{2} \geq 25 \%$ while mechanically ventilated or an $\mathrm{FiO}_{2} \geq 35 \%$ while on noninvasive respiratory support at day 14 of life. ${ }^{30}$

\section{Endothelial Progenitor Cells-The Vascular Hypothesis of BPD}

\section{Definition}

The term endothelial progenitor cell (EPC) is used to describe a cell that can regenerate the endothelial lining of blood 
vessels. As for other stem cells, the nomenclature remains ambiguous, leading to controversy in interpreting study results. A recent consensus statement on nomenclature of EPCs suggests the use of precise terminology based on defining cellular phenotype and function. ${ }^{31}$ Two distinct and well-defined cell types have been considered as EPCs: endothelial colony forming cells (ECFCs) and myeloid angiogenic cells (MACs). Both promote vascular repair and may thus be considered for therapeutic purposes, although their mechanisms of action appear to be different. ECFCs are characterized by (1) robust proliferative potential, (2) secondary and tertiary colony formation upon replating, and (3) de novo blood vessel formation in vivo when transplanted into immunodeficient mice. Of clinical relevance for neonatology, these cells exist in cord blood and can be expanded for therapeutic purposes. ${ }^{32}$ MACs also participate in angiogenesis but do not directly form the endothelial monolayer of new vessels and display various monocyte/macrophage phenotypes and function.

\section{Rationale and Biological Plausibility}

Increasing evidence suggests that lung vascular growth and angiogenic growth factors are crucial for lung growth and repair. ${ }^{33}$ Interestingly, resident ECFCs are present in the developing human and rat lung ${ }^{34}$ and their function is impaired in experimental neonatal lung injury. ${ }^{35}$ Combining the data from a recent systematic review indicating a link between impaired EPC function in circulating/cord blood of preterm infants and preterm birth-associated complications $^{36}$ suggests that exogenous supplementation of EPCs may be therapeutic. Unlike MSCs, however, ECFCs may be immunogenic, thus requiring autologous transplantation.

\section{Preclinical Evidence}

The first observation with bone marrow-derived MACs showed restoration of alveolar and lung vascular growth in hyperoxic neonatal mice. ${ }^{37}$ In another study, short-term cultured bone marrow-derived cells with "EPC characteristics" lead to partial recovery in alveolar septal number in this same animal model. ${ }^{38}$ Short-term cultured EPCs impaired alveolar growth in normoxic pups and longterm cultured cells caused some aberrant tissue growth raising the importance of safety and long-term studies in the preclinical setting.

IV infusion of human cord blood-derived ECFCs into immune-deficient rats and mice exposed to hyperoxia promotes alveolar and lung vascular growth, and attenuates pulmonary hypertension. ${ }^{35}$ These effects persist after 10 months and no tumor formation was noted. Surprisingly, ECFCs do not engraft and seem to act through a paracrine effect since the cell-free ECFC-derived conditioned media exert similar benefit to whole cell therapy. ${ }^{35}$ In a bleomycininduced neonatal lung injury model, ECFC-derived conditioned media had no effect on lung growth but attenuated $\mathrm{pH}^{39}$

Focused preclinical studies are required before clinical translation of this cell type can be considered in neonates.

\section{CD34 $^{+}$Mononuclear Cells-The Old Fashioned, Straight from the Bone Marrow Transplant Literature}

\section{Definition}

Hematopoietic stem cell (HSC) transplantation has been practiced for over 50 years in cancer patients to reconstitute bone marrow function. The consideration of cell therapy for regenerative indications is new.

\section{Rationale and Biological Plausibility}

Within HSCs, CD34 ${ }^{+}$represents a marker for engraftment. Earlier studies suggested some lung engraftment and differentiation of bone marrow-derived cells. ${ }^{40}$ These observations have now been dispelled ${ }^{41}$ and the rationale for using these mononuclear cells (MNCs) should be reexamined.

\section{Preclinical Evidence}

Four studies have explored the lung protective effect of MNCs. ${ }^{42-45}$ Intranasal inoculation of fresh human cord blood CD34 ${ }^{+}$MNCs into neonatal mice with apoptosis-induced lung injury resulted in sparse engraftment and very limited alveolar epithelial differentiation yet improved lung growth 1 year after transplantation. IV injection of MNCs from cryopreserved cord blood into hyperoxia-exposed newborn mice significantly attenuated methacholine-induced airway hyperreactivity, and mildly improved alveolarization, lung compliance, and elastance. Interestingly, total nucleated cells had no effect and granular cells caused high mortality and an emphysematous phenotype. ${ }^{46}$ Intraperitoneal injection of human umbilical cord blood MNCs in a double-hit mouse model combining antenatal hypoxia and postnatal hyperoxia improved septal thickness and decreased Tgf $\beta 3$ mRNA expression and proinflammatory IL-1 $\beta$. Finally, intratracheal injection of cord blood MNCs in neonatal hyperoxic rats had no effect on alveolarization and lower anti-inflammatory and no angiogenic effects compared with cord blood-derived MSCs or human adipose tissue-derived MSCs. ${ }^{47}$ This study highlights an interesting debate about therapeutic potency of various cell therapies.

\section{Human Amniotic Fluid Stem Cells-Don't Throw Away the Bath Water}

\section{Definition}

The discovery of cells in the amniotic fluid displaying stem cell characteristics provided yet another potential perinatal source for therapeutic cells. These human amniotic fluid stem cells (hAFSCs) express embryonic and adult stem cell markers, expand extensively without feeders, and are multipotent as they can differentiate into cell types representing each embryonic germ layer, including cells of adipogenic, osteogenic, myogenic, endothelial, neuronal, and hepatic lineages.

\section{Rationale and Biological Plausibility}

Previous literature suggests anti-inflammatory effects of hAFSCs ${ }^{48}$ as well as the ability to integrate and differentiate into epithelial lung lineages. ${ }^{49}$ 


\section{Preclinical Evidence}

One study so far explored the lung protective effect of hAFSCs. ${ }^{50}$ Intratracheal injection after established hyperoxic lung injury in neonatal rats showed low hAFSC retention, improved alveolar and lung vascular structure as well as increased vascular endothelial growth factor expression, suggesting yet again-despite their multipotency-a paracrine mechanism of action. Given the ease of access to other perinatal sources such as cord, cord blood, and placenta, it remains to be tested if hAFSCs provide superior repair properties than MSCs and hAECs.

\section{Induced Pluripotent Stem Cells-Personalized Cell Therapy}

\section{Definition}

Induced pluripotent stem cells (iPSCs) are differentiated cells that have been genetically reprogrammed to an embryonic stem cell (ESC)-like state by forced expression of a combination of four transcription factors (Oct3/4, Sox2, Klf4, and c-Myc) important for maintaining the defining properties of ESCs. ${ }^{51,52}$

iPSCs possess desirable characteristics for use as a cell therapy. Like ESCs, iPSCs have the potential to become any cell type in the body. Increasingly robust methods of differentiation into alveolar epithelial type 2 cells (AT2) are described. ${ }^{53}$ Human iPSCs (hiPSCs) can be generated from each patient, thus representing an autologous source of cells that overcome the likelihood of immune-mediated rejection. An unlimited number of cells can be generated from hiPSCs as they can be maintained and expanded in vitro.

\section{Rationale and Biological Plausibility}

AT2s, a subset of which represent distal lung progenitor cells, promote normal lung growth and repair after injury. ${ }^{42}$ AT2 depletion is postulated to contribute to persistent lung injury in BPD. ${ }^{42-45}$

\section{Preclinical Evidence}

One study so far explored the lung-protective effect of iPSCderived AT2s. Proof-of-concept airway delivery of primary murine AT2s prevent hyperoxia-induced impairment in lung function and alveolar growth in neonatal mice. ${ }^{54}$ Undifferentiated murine and human (h)iPSCs also preserve lung function and alveolar growth but cause local teratoma formation and systemic cellular infiltration in various organs. Conversely, airway delivery of hiPSC-derived AT2 improves lung function and structure without evidence of tumor formation at 8 months and some degree of engraftment. Further studies need to confirm the mechanism of action and importantly, the safety of differentiated iPSCs. The pace of advances in the iPSC field is impressive-as indicated by the recent description of fail-safe iPSCs $^{55}$-and the ancillary knowledge generated from iPSC biology may lead to a better understanding of organ regeneration.

Overall, these observations indicate stem cell-based therapies as a growing field of investigation with MSCs and hAECs as the front runners since they have entered early-phase clinical trials. A common denominator of all these putative cell pro- ducts-independent of the original rationale and biological plausibility for using these cells in the first place-is the lack of engraftment and the absence of potency assays to predict therapeutic efficacy in vivo before infusing the product.

\section{Knowledge Gaps}

\section{Cell Manufacturing}

Clinical-grade cell products have to be manufactured according to good manufacturing practices to ensure a consistent and controlled product according to quality standards. Since the "process is the product," each step along the manufacturing process will affect the quality and thus the therapeutic potency of the product. ${ }^{56}$

For example, preconditioning of bone marrow MSCs by ex vivo exposure to hyperoxia prior to intratracheal injection enhances the lung protective effect in neonatal hyperoxiainduced lung injury in rats. ${ }^{57}$ Interestingly, female bone marrow-derived MSCs seem to have greater therapeutic efficacy than male MSCs on lung inflammation and vascular remodeling in this same animal model and this effect is more pronounced in male animals. ${ }^{58}$ Conversely, exposure to surfactant reduces MSC viability and combined MSC + surfactant administration does not exert additive lung protective effects on lung structure in hyperoxia-induced lung injury. ${ }^{59}$

These findings indicate that much more needs to be learned about the biology and manufacturing of cell-based therapies, starting with the cell source (adult bone marrow, cord blood, cord tissue, placenta, etc.), isolation-cultureexpansion techniques, cryopreservation protocols, the choice of using a frozen versus a fresh cell product, an autologous versus an allogeneic strategy, the cell type, or a cell versus a cell-free product. ${ }^{60}$

Indeed, one exciting new avenue is the recognition that MSCs exert their therapeutic benefit through the release of EVs. Amongst these EVs, exosomes, membrane-derived nano-sized particles that contain proteins and miRNA, have attracted particular attention. ${ }^{61}$ Exosomes perform as well as if not better in protecting the lung from hyperoxic injury. ${ }^{9,62-64}$ Rigorous preclinical studies need to confirm the initial results of these exploratory studies. However, similar to the actual cell product, manufacturing at the clinical scale and prediction of the bioactivity remain a challenge for the implementation of these "nanotherapies for micropreemies." 65

\section{Potency Assays}

An ideal scenario can be found in the HSC field where CD34 represents a marker for engraftment and thus a potential indicator of successful replenishment of bone marrow function. Unfortunately, cell therapies in regenerative medicine still lack reliable and rapid disease- and mechanism-specific in vitro assays to predict their therapeutic efficacy. In vivo tests are still lacking. Recent advances in 3D lung organoids that replicate an organ-in-a-dish may yield more reliable results than traditional culture techniques. ${ }^{66}$ The most promising approach today is the CLinical Indications Prediction (CLIP) scale to predict how donor-to-donor heterogeneity and culture conditions impact the therapeutic efficacy of $\mathrm{MSCs}^{67}$ : 
high Twist1 levels predict proangiogenic properties, while low Twist1 levels predict a more anti-inflammatory activity. Combined with omic technology, a multipanel of factors may achieve accurate prediction of the potency of a given cell product which in turn would substantially benefit our understanding of the mechanism of action and facilitate the manufacturing process.

\section{Conclusion}

MSC and hAEC-based therapies have entered early-phase clinical trials in neonatology. Numerous other cell types are being investigated in the laboratory. Nonetheless, these are early days for cell therapy and major gaps in our knowledge persist with regard to the mechanism of action of these various cells. Strong rationale and biological plausibility need to be confirmed in rigorous preclinical studies. Potency assays need to be established to predict in vivo bioactivity and to facilitate and fine-tune the manufacturing process of a given cell product. Well-designed clinical trials-based on robust preclinical evaluation-will permit successful (safe and timely) translation of these potentially game-changing therapies in neonatology.

\section{Conflict of Interest}

None declared.

\section{References}

1 Stoll BJ, Hansen NI, Bell EF, et al; Eunice Kennedy Shriver National Institute of Child Health and Human Development Neonatal Research Network. Trends in care practices, morbidity, and mortality of extremely preterm neonates, 1993-2012.JAMA 2015;314 (10):1039-1051

2 Owen LS, Manley BJ, Davis PG, Doyle LW. The evolution of modern respiratory care for preterm infants. Lancet 2017;389 (10079):1649-1659

3 Thebaud B. Chronic lung disease in the neonate: past, present, and future. Neoreviews 2013;14(05):e252-e258

4 Collaco JM, McGrath-Morrow SA. Respiratory phenotypes for preterm infants, children, and adults: bronchopulmonary dysplasia and more. Ann Am Thorac Soc 2018;15(05):530-538

5 Goss KN, Beshish AG, Barton GP, et al. Early Pulmonary Vascular Disease in Young Adults Born Preterm. American Journal of Respiratory and Critical Care Medicine 2018;198(12):1549-1558

6 Heinonen K, Eriksson JG, Lahti J, et al. Late preterm birth and neurocognitive performance in late adulthood: a birth cohort study. Pediatrics 2015;135(04):e818-e825

7 Fischbach MA, Bluestone JA, Lim WA. Cell-based therapeutics: the next pillar of medicine. Sci Transl Med 2013;5(179):179ps7

8 Fung ME, Thébaud B. Stem cell-based therapy for neonatal lung disease: it is in the juice. Pediatr Res 2014;75(1-1):2-7

9 Willis GR, Fernandez-Gonzalez A, Anastas J, et al. Mesenchymal stromal cell exosomes ameliorate experimental bronchopulmonary dysplasia and restore lung function through macrophage immunomodulation. Am J Respir Crit Care Med 2018;197(01):104-116

10 Dominici M, Le Blanc K, Mueller I, et al. Minimal criteria for defining multipotent mesenchymal stromal cells. The International Society for Cellular Therapy position statement. Cytotherapy 2006;8(04):315-317

11 Friedenstein AJ, Gorskaja JF, Kulagina NN. Fibroblast precursors in normal and irradiated mouse hematopoietic organs. Exp Hematol 1976;4(05):267-274
12 Crisan M, Yap S, Casteilla L, et al. A perivascular origin for mesenchymal stem cells in multiple human organs. Cell Stem Cell 2008;3(03):301-313

13 Popova AP, Bozyk PD, Bentley JK, et al. Isolation of tracheal aspirate mesenchymal stromal cells predicts bronchopulmonary dysplasia. Pediatrics 2010;126(05):e1127-e1133

14 Möbius MA, Freund D, Vadivel A, et al. Oxygen disrupts human fetal lung mesenchymal cells. Implications for bronchopulmonary dysplasia. Am J Respir Cell Mol Biol 2019;60(05):592-600

15 Collins JJP, Lithopoulos MA, Dos Santos CC, et al. Impaired angiogenic supportive capacity and altered gene expression profile of resident $\mathrm{CD} 146^{+}$mesenchymal stromal cells isolated from hyperoxia-injured neonatal rat lungs. Stem Cells Dev 2018; 27(16):1109-1124

16 Aslam M, Baveja R, Liang OD, et al. Bone marrow stromal cells attenuate lung injury in a murine model of neonatal chronic lung disease. Am J Respir Crit Care Med 2009;180(11):11221130

17 van Haaften T, Byrne R, Bonnet S, et al. Airway delivery of mesenchymal stem cells prevents arrested alveolar growth in neonatal lung injury in rats. Am J Respir Crit Care Med 2009;180 (11):1131-1142

18 Pierro M, Ionescu L, Montemurro T, et al. Short-term, long-term and paracrine effect of human umbilical cord-derived stem cells in lung injury prevention and repair in experimental bronchopulmonary dysplasia. Thorax 2013;68(05):475-484

19 Chang YS, Choi SJ, Ahn SY, et al. Timing of umbilical cord blood derived mesenchymal stem cells transplantation determines therapeutic efficacy in the neonatal hyperoxic lung injury. PLoS One 2013;8(01):e52419

20 Augustine S, Avey MT, Harrison B, et al. Mesenchymal stromal cell therapy in bronchopulmonary dysplasia: systematic review and meta-analysis of preclinical studies. Stem Cells Transl Med 2017;6 (12):2079-2093

21 Chang YS, Ahn SY, Yoo HS, et al. Mesenchymal stem cells for bronchopulmonary dysplasia: phase 1 dose-escalation clinical trial. J Pediatr 2014;164(05):966.e6-972.e6

22 Ahn SY, Chang YS, Kim JH, Sung SI, Park WS. Two-year follow-up outcomes of premature infants enrolled in the phase I trial of mesenchymal stem cells transplantation for bronchopulmonary dysplasia. J Pediatr 2017;185:49-54.e2

23 Álvarez-Fuente M, Arruza L, Lopez-Ortego P, et al. Off-label mesenchymal stromal cell treatment in two infants with severe bronchopulmonary dysplasia: clinical course and biomarkers profile. Cytotherapy 2018;20(11):1337-1344

24 Pierro M, Thébaud B, Soll R. Mesenchymal stem cells for the prevention and treatment of bronchopulmonary dysplasia in preterm infants. Cochrane Database Syst Rev 2017;11:CD011932

25 Ilancheran S, Michalska A, Peh G, Wallace EM, Pera M, Manuelpillai U. Stem cells derived from human fetal membranes display multilineage differentiation potential. Biol Reprod 2007;77(03): 577-588

26 Vosdoganes P, Hodges RJ, Lim R, et al. Human amnion epithelial cells as a treatment for inflammation-induced fetal lung injury in sheep. Am J Obstet Gynecol 2011;205(02):156.e26-156.e33

27 Vosdoganes P, Lim R, Koulaeva E, et al. Human amnion epithelial cells modulate hyperoxia-induced neonatal lung injury in mice. Cytotherapy 2013;15(08):1021-1029

28 Vosdoganes P, Wallace EM, Chan ST, Acharya R, Moss TJ, Lim R. Human amnion epithelial cells repair established lung injury. Cell Transplant 2013;22(08):1337-1349

$29 \operatorname{Lim}$ R, Malhotra A, Tan J, et al. First-in-human administration of allogeneic amnion cells in premature infants with bronchopulmonary dysplasia: a safety study. Stem Cells Transl Med 2018;7 (09):628-635

30 Baker EK, Malhotra A, Lim R, et al. Human amnion cells for the prevention of bronchopulmonary dysplasia: a protocol for a phase I dose escalation study. BMJ Open 2019;9(02):e026265 
31 Medina RJ, Barber CL, Sabatier F, et al. Endothelial progenitors: a consensus statement on nomenclature. Stem Cells Transl Med 2017;6(05):1316-1320

32 Ingram DA, Mead LE, Tanaka H, et al. Identification of a novel hierarchy of endothelial progenitor cells using human peripheral and umbilical cord blood. Blood 2004;104(09):2752-2760

33 Thébaud B, Abman SH. Bronchopulmonary dysplasia: where have all the vessels gone? Roles of angiogenic growth factors in chronic lung disease. Am J Respir Crit Care Med 2007;175(10):978-985

34 Alphonse RS, Vadivel A, Zhong S, et al. The isolation and culture of endothelial colony-forming cells from human and rat lungs. Nat Protoc 2015;10(11):1697-1708

35 Alphonse RS, Vadivel A, Fung M, et al. Existence, functional impairment, and lung repair potential of endothelial colony-forming cells in oxygen-induced arrested alveolar growth. Circulation 2014;129 (21):2144-2157

36 Bertagnolli M, Nuyt AM, Thébaud B, Luu TM. Endothelial progenitor cells as prognostic markers of preterm birth-associated complications. Stem Cells Transl Med 2017;6(01):7-13

37 Balasubramaniam V, Ryan SL, Seedorf GJ, et al. Bone marrowderived angiogenic cells restore lung alveolar and vascular structure after neonatal hyperoxia in infant mice. Am J Physiol Lung Cell Mol Physiol 2010;298(03):L315-L323

38 Firsova AB, Bird AD, Abebe D, Ng J, Mollard R, Cole TJ. Fresh noncultured endothelial progenitor cells improve neonatal lung hyperoxia-induced alveolar injury. Stem Cells Transl Med 2017;6 (12):2094-2105

39 Baker CD, Seedorf GJ, Wisniewski BL, et al. Endothelial colony-forming cell conditioned media promote angiogenesis in vitro and prevent pulmonary hypertension in experimental bronchopulmonary dysplasia. Am J Physiol Lung Cell Mol Physiol 2013;305(01):L73-L81

40 Krause DS, Theise ND, Collector MI, et al. Multi-organ, multilineage engraftment by a single bone marrow-derived stem cell. Cell 2001;105(03):369-377

41 Kotton DN, Fabian AJ, Mulligan RC. Failure of bone marrow to reconstitute lung epithelium. Am J Respir Cell Mol Biol 2005;33 (04):328-334

42 Desai TJ, Brownfield DG, Krasnow MA. Alveolar progenitor and stem cells in lung development, renewal and cancer. Nature 2014; 507(7491):190-194

43 Reddy R, Buckley S, Doerken M, et al. Isolation of a putative progenitor subpopulation of alveolar epithelial type 2 cells. Am J Physiol Lung Cell Mol Physiol 2004;286(04):L658-L667

44 Chapman HA, Li X, Alexander JP, et al. Integrin $\alpha 6 \beta 4$ identifies an adult distal lung epithelial population with regenerative potential in mice. J Clin Invest 2011;121(07):2855-2862

45 Lin SE, Barrette AM, Chapin C, et al. Expression of human carcinoembryonic antigen-related cell adhesion molecule 6 and alveolar progenitor cells in normal and injured lungs of transgenic mice. Physiol Rep 2015;3(12):e12657

46 Monz D, Tutdibi E, Mildau C, et al. Human umbilical cord blood mononuclear cells in a double-hit model of bronchopulmonary dysplasia in neonatal mice. PLoS One 2013;8(09):e74740

47 Ahn SY, Chang YS, Sung DK, et al. Cell type-dependent variation in paracrine potency determines therapeutic efficacy against neonatal hyperoxic lung injury. Cytotherapy 2015;17(08):1025-1035

48 Moorefield EC, McKee EE, Solchaga L, et al. Cloned, CD117 selected human amniotic fluid stem cells are capable of modulating the immune response. PLoS One 2011;6(10):e26535

49 Carraro G, Perin L, Sedrakyan S, et al. Human amniotic fluid stem cells can integrate and differentiate into epithelial lung lineages. Stem Cells 2008;26(11):2902-2911
50 Grisafi D, Pozzobon M, Dedja A, et al. Human amniotic fluid stem cells protect rat lungs exposed to moderate hyperoxia. Pediatr Pulmonol 2013;48(11):1070-1080

51 Takahashi K, Tanabe K, Ohnuki M, et al. Induction of pluripotent stem cells from adult human fibroblasts by defined factors. Cell 2007;131(05):861-872

52 Takahashi K, Yamanaka S. Induction of pluripotent stem cells from mouse embryonic and adult fibroblast cultures by defined factors. Cell 2006;126(04):663-676

53 Jacob A, Morley M, Hawkins F, et al. Differentiation of human pluripotent stem cells into functional lung alveolar epithelial cells. Cell Stem Cell 2017;21(04):472.e10-488.e10

54 Shafa M, Ionescu LI, Vadivel A, et al. Human induced pluripotent stem cell-derived lung progenitor and alveolar epithelial cells attenuate hyperoxia-induced lung injury. Cytotherapy 2018;20 (01):108-125

55 Liang Q Monetti C, Shutova MV, et al. Linking a cell-division gene and a suicide gene to define and improve cell therapy safety. Nature 2018;563(7733):701-704

56 Martin I, De Boer J, Sensebe L; MSC Committee of the International Society for Cellular Therapy. A relativity concept in mesenchymal stromal cell manufacturing. Cytotherapy 2016;18 (05):613-620

57 Waszak P, Alphonse R, Vadivel A, Ionescu L, Eaton F, Thébaud B. Preconditioning enhances the paracrine effect of mesenchymal stem cells in preventing oxygen-induced neonatal lung injury in rats. Stem Cells Dev 2012;21(15):2789-2797

58 Sammour I, Somashekar S, Huang J, et al. The effect of gender on mesenchymal stem cell (MSC) efficacy in neonatal hyperoxia-induced lung injury. PLoS One 2016;11(10): e0164269

59 Chen CM, Chou HC, Lin W, Tseng C. Surfactant effects on the viability and function of human mesenchymal stem cells: in vitro and in vivo assessment. Stem Cell Res Ther 2017;8(01):180

60 Chinnadurai R, Copland IB, Garcia MA, et al. Cryopreserved mesenchymal stromal cells are susceptible to t-cell mediated apoptosis which is partly rescued by IFN $\gamma$ licensing. Stem Cells 2016;34(09):2429-2442

61 Kourembanas S. Exosomes: vehicles of intercellular signaling, biomarkers, and vectors of cell therapy. Annu Rev Physiol 2015; 77:13-27

62 Bryan C, Sammour I, Guerra K, et al. TNF $\alpha$-stimulated protein 6 (TSG-6) reduces lung inflammation in an experimental model of bronchopulmonary dysplasia. Pediatr Res 2019;85 (03):390-397

63 Lal CV, Olave N, Travers C, et al. Exosomal microRNA predicts and protects against severe bronchopulmonary dysplasia in extremely premature infants. JCI Insight 2018;3(05):93994

64 Porzionato A, Zaramella P, Dedja A, et al. Intratracheal administration of clinical-grade mesenchymal stem cell-derived extracellular vesicles reduces lung injury in a rat model of bronchopulmonary dysplasia. Am J Physiol Lung Cell Mol Physiol 2019;316(01):L6-L19

65 Lesage F, Thébaud B. Nanotherapies for micropreemies: stem cells and the secretome in bronchopulmonary dysplasia. Semin Perinatol 2018;42(07):453-458

66 Sucre JMS, Jetter CS, Loomans H, et al. Successful establishment of primary type II alveolar epithelium with 3D organotypic coculture. Am J Respir Cell Mol Biol 2018;59(02):158-166

67 Boregowda SV, Krishnappa V, Haga CL, Ortiz LA, Phinney DG. A clinical indications prediction scale based on TWIST1 for human mesenchymal stem cells. EBioMedicine 2015;4:62-73 